\section{More weapons in the arsenal against ischemic retinopathy}

\author{
Eli Keshet \\ Department of Molecular Biology, The Hebrew University-Hadassah Medical School, \\ Jerusalem 91120, Israel. Phone: (972) 2-6758496, Fax: 972-2-6757195; E-mail: keshet@cc.huji.ac.il.
}

\section{Commentary}

See related article, Volume 107, Number 6, pages 717-725.
Ischemia-induced retinopathy is the major cause of blindness world-wide. Diverse processes may lead to retinal ischemia, including a central retinal vein occlusion, a progressive deterioration of vascular function due to prolonged hyperglycemia, and obliteration of newly formed retinal vessels in premature neonates subjected to hyperoxia. The later two processes may culminate in the development of proliferative diabetic retinopathy (PDR) and retinopathy of prematurity (ROP), respectively. While different etiologies may lead to insufficient perfusion of the retina, once a critical level of ischemia has been achieved, the different forms of retinopathy seem to converge on a common sequela - the hypoxia-induced formation of morphologically abnormal vessels. Retinopathy-associated neovascularization is excessive and neo-vessels are abnormally leaky and tend to breach the inner limited membrane of the retina and invade the vitreous. Such preretinal vascular growth is perhaps the most devastating aspect of retinopathy, as it often leads to retina detachment and eventually to blindness.

Combating retinopathy can, in principle, be directed against the first pathogenic phase, the generation of a vascular deficit and the resultant ischemia, or against the second phase, the formation of surplus, abnormal vessels. At least for PDR, the former approach has been impossible because we lack sufficient insight into the mechanisms leading to progressive vessel dysfunction, or even a suitable animal model in which to study these events. For this reason, most of the studies outlined below have resorted to the use of an oxygeninduced retinopathy model (i.e., an ROP model) (1).

\section{VEGF and retinal neovascularization}

The concept that retinopathy-associated neovascularization is driven by a hypoxia-induced angiogenic factor was proposed over half a century ago. More recent evidence that VEGF is the key mediator of angiogenic feedback responses to hypoxia led to the identification of VEGF as what Michaelson, in his 1948 paper (2) termed "factor X." Many studies have indeed shown that VEGF is strongly upregulated in the ischemic retina and may also leak and accumulate in the vitreous of retinopathy patients (3). Justly, VEGF has become the primary target for ischemia-induced retinopathy. Several modes of counteracting VEGF were proven effective in animal ROP models, including the use of antisense oligonucleotides, administration of VEGF-neutralizing antibodies, the use of a soluble VEGF receptor chimeric protein, and the inhibition of VEGF signaling using specific kinase inhibitors $(4,5)$. Kinase inhibitors with a broader specificity have also been examined in hopes of blocking other factors that cooperate with VEGF in promoting angiogenesis. Notably, Seo et al. found that a kinase inhibitor that blocks phosphorylation of VEGF, PDGF, and several protein kinase $\mathrm{C}$ isoforms completely inhibits retinal neovascularization (6).

For several reasons, targeting VEGF might be problematic as a therapy for retinal neovascularization. VEGF is a multifunctional factor that, among other activities, serves as a survival factor for retinal vessels (7). The fact that VEGF is constitutively produced by the retinal pigmented epithelium of the normal eye, for example, presumably attests for a role of VEGF in vascular maintenance. Furthermore, the chronic nature of PDR and the intrinsic risk of repeated intraocular injections might necessitate a prolonged systemic administration of VEGF antagonists, which could adversely affect other organ systems. Accordingly, alternative approaches have been attempted, targeting different components of the angiogenic cascade downstream of VEGF. In an ROP model, inhibitors of matrix metalloproteinases, blocking antibodies directed at specific integrins implicated in angiogenesis, and an NF- $\kappa \mathrm{B}$ inhibitor (8-10), as well as natural angiogenesis inhibitors, such as thrombospondin-1 and the recently discovered pigment epithelium-derived factor (PEDF), have all been applied to ischemia-induced retinopathy with some success $(11,12)$. An obvious advantage of these inhibitors is that they can block neovascularization induced by multiple factors. Indeed, systemically delivered PEDF completely blocked pre-retinal growth at a relatively low dose (12). Efforts are continuing to further extend the arsenal of antiangiogenic agents that could be used either alone or in combination to attenuate ischemiainduced retinopathy.

\section{Distinct roles for eNOS and iNOS}

In a recent issue of the JCI, Sennlaub et al. proposed the use of nitric oxide synthase (NOS) inhibitors as agents to block pre-retinal growth (13). Like VEGF, NO production is induced by hypoxia in an HIF-1-dependent manner. Thus, VEGF and NO are coinduced in the same ischemic regions of the retina and elevated levels of both are detectable in the vitreous of PDR patients. A number of studies have demonstrated an intricate interplay between VEGF and NO: NO is required downstream of VEGF in a pathway leading to endothelial cell proliferation and angiogenesis $(14,15)$. In addition, VEGF also stimulates production of NO by endothelial cells $(15,16)$ and $\mathrm{NO}$, in turn, can block hypoxia-induced production of VEGF (17) as well as its induction following vascular injury (18). This reciprocal relation between VEGF and NO has suggested a negative feedback loop to downregulate VEGF expression to basal levels. Sennlaub et al. made use of iNOS-null mice to examine the effects of iNOS deficiency at different stages in the development of ischemia-induced retinopathy. iNOS deficiency did not affect the early step of hyperoxia-induced vessel obliteration. Interestingly, however, Brooks et 
al. (19) recently found that eNOS deficiency (or, alternatively, its pharmacological inhibition) protects the retina from vessel obliteration, presumably by reducing levels of NO-derived oxidants. Modulation of eNOS activity may therefore be of use in preventing ROP (19). iNOS deficiency, however, accelerates revascularization of the avascular retina and significantly reduces vitreal invasion and pre-retinal growth. A specific iNOS inhibitor administered immediately after the hyperoxic insult gave similar results, albeit yielding only a modest attenuation of pre-retinal vessel growth (13).

\section{Prospects for new therapies}

Because pre-retinal growth is the most damaging aspect of retinopathy, this poorly understood event is an attractive therapeutic target. Previous studies have shown that breaching the inner limiting membrane of the retina takes place following hypoxia-induced degeneration of astrocytes, which normally serve as scaffolds for vessels spreading on the retina surface (20). Other studies (12) have suggested that vitreal invasion takes place upon downregulation of natural angiogenic inhibitors residing in the vitreous. PEDF, in particular, prevents vessel invasion into avascular compartments of the eye like the vitreous, cornea, and outer nuclear layer of the retina. Interestingly, VEGF and PEDF are regulated by oxygen in an opposite manner. The study of Sennlaub et al. is consistent with the thesis that pre-retinal growth is elicited whenever the failure to restore retinal normoxia results in a continuous build-up of VEGF in the vitreous (13). Taken together, these two new studies strongly suggest that the balance of pro- and antiangiogenic activities in the vitreous determine the extent of pre-retinal growth.

Vitreal neovascularization shows different characteristics from intraretinal neovascularization. The remarkably dif- ferent architecture of vitreal vessels presumably reflect some qualitative differences - perhaps an altered engagement with periendothelial cells or a less mature phenotype - that might be exploited to obliterate the vitreal vessels selectively, rather than through generalized antiangiogenic approaches. Another approach might take advantage of the differential accessibility of vitreal vessels to certain vectors delivered intraocularly.

In summary, a growing number of different therapeutic modalities have been explored to inhibit vasculopathic growth in ischemic retinopathy. While some of these agents are currently in or approaching clinical trials, certain concerns remain. Notably, most agents have been tested in a rodent model of oxygen-induced retinopathy, where retinal ischemia and ensuing neovascularization are induced within a few days. This is in sharp contrast to the more frequent and serious case of PDR that develops over a period of years, which might require repeated treatments over an extended period of time. Furthermore, the premise that the abnormal features of vessels induced by a short hyperoxic episode in the immature retina are qualitatively identical to those induced by prolonged hyperglycemia in the ageing retina needs to be substantiated. Nonetheless, the growing number of agents that can block ischemic retinopathy increase the hope for new therapies that will be more effective than current treatments in the battle against retinopathies and the blindness they cause.

1. Smith, L.E, et al. 1994. Oxygen-induced retinopathy in the mouse. Invest. Ophthalmol. Vis. Sci. 35:101-111.

2. Michaelson, I.C. 1948. The mode of development of the vascular system of the retina with some observations on its significance for certain retina diseases. Trans. Ophthalmol. Soc. U.K. 68:137-180

3. Aiello, L.P. 1994. Vascular endothelial growth factor in ocular fluid of patients with proliferative diabetic retinopathy and other retinal disorders. N. Engl. J. Med. 331:1480-1487.

4. Robinson, G.S., et al. 1996. Oligodeoxynucleotides inhibit retinal neovascularization in a murine model of proliferative retinopathy. Proc.
Natl. Acad. Sci. USA. 93:4851-4856.

5. Aiello, L.P., et al. 1995. Suppression of retinal neovascularization in vivo by inhibition of VEGF using soluble VEGF-receptor chimeric proteins. Proc. Natl. Acad. Sci. USA. 92:10457-10461.

6. Seo, M.S., et al. 1999. Dramatic inhibition of retinal and choroidal neovascularization by oral administration of a kinase inhibitor. Am. J. Pathol. 54:1743-1753.

7. Alon, T., et al. 1995. Vascular endothelial growth factor acts as a survival factor for newly formed retinal vessels and implications for retinopathy of prematurity. Nat. Med. 1:1024-1028.

8. Das, A., McLamore, A., and McGuire, P.G. 1999 Retinal neovascularization is suppressed with a matrix metalloproteinase inhibitor. Arch. Ophthalmol. 117:498-503.

9. Friedlander, M., et al. 1996. Involvement of integrins $\alpha v \beta 3$ and $\alpha v \beta 5$ in ocular neovascular diseases. Proc. Natl. Acad. Sci. USA. 93:9764-9769.

10. Yoshida, A., Yoshida, S., Ishibashi, T., Kuwano, M., and Inomata, H. 1999. Suppression of retinal neovascularization by the NF-kappaB inhibitor pyrrolidone dithiocarbamate in mice. Invest. $O p h$ thalmol. Vis. Sci. 40:1624-1629.

11. Shafiee, A., et al. 2000. Inhibition of retinal angiogenesis by peptides derived from thrombospondin1. Invest. Ophthalmol. Vis. Sci. 41:2378-2388.

12. Stellmach, V., Crawford, S.E., Zhou, W., and Bouck, N. 2001. Prevention of ischemia-induced retinopathy by the natural ocular antiangiogenic agent pigment epithelium-derived factor. Proc. Natl. Acad. Sci. USA. 98:2593-2597.

13. Sennlaub, F., Courtois, Y., and Goureau, O. 2001 Inducible nitric oxide synthase mediates the change from retinal to vitreal neovascularization in ischemic retinopathy. J. Clin. Invest. 107:717-725.

14. Ziche, M., et al. 1997. Nitric oxide synthase lies downstream from VEGF-induced but not basic fibroblast growth factor-induced angiogenesis. J. Clin. Invest. 99:2625-2634.

15. Papapetropoulos, A., Garcia-Cardena, G., Madri, J.A., and Sessa, W.C. 1997. Nitric oxide production contributes to angiogenic properties of vascular endothelial growth factor in human endothelial cells. J. Clin. Invest. 100:3131-3139.

16. Hood, J.D., Meininger, C.J., Ziche, M., and Granger, H.J. 1998. VEGF upregulates ecNOS message, protein, and NO production in human endothelial cells. Am. J. Physiol. 274:H1054-H1058.

17. Ghiso, N., Rohan, R.M., Amano, S., Gerland, R and Adamis, A.P. 1999. Suppression of hypoxiaassociated vascular endothelial growth factor gene expression by nitric oxide via cGMP. Invest. Ophthalmol. Vis. Sci. 40:1033-1039.

18. Tsurumi, Y., et al. 1997. Reciprocal relation between VEGF and NO in the regulation of endothelial integrity. Nat. Med. 3:879-886.

19. Brooks, S.E., et al. 2001. Reduced severity of oxygen-induced retinopathy in eNOS-deficient mice. Invest. Ophthalmol. Vis. Sci. 42:222-228.

20. Stone, J., et al. 1996. Roles of vascular endothelial growth factor and astrocytes degeneration in the genesis of retinopathy of prematurity. Invest. Oph thalmol. Vis. Sci. 37:290-299. 